\title{
MEASUREMENT OF BLOOD FLOW IN THE COLLATERAL VESSELS OF THE PORTAL VEIN; PRELIMINARY RESULTS OF A NEW METHOD
}

\author{
By F. L. IBER,* D. N. S. KERR, W. DÖLLE and S. SHERLOCK \\ (From the Department of Medicine, Postgraduate Medical School of the University of \\ London, England)
}

(Submitted for publication September 18, 1959; accepted April 7, 1960)

The portal vein and its branches may be demonstrated by the intrasplenic injection of radiocontrast media. Such identification of collateral vessels aids understanding of some of the symptoms of chronic liver disease $(1-4)$. The size and location of collateral vessels is shown; however, the blood flow through them is not measured. Certain syndromes, such as the neurological complications of chronic liver disease (portal systemic encephalopathy), depend more on the total collateral blood flow than on the presence of any specific collateral vessels (5). This paper reports a method for measuring the portal collateral blood flow and its application in 12 patients.

If an indicator substance is introduced into, and is rapidly absorbed from the spleen, part of it reaches the general circulation by passing through the liver and the remainder by passing through any collateral channels present (Figure 1). The amount passing by each of the two routes is directly proportional to its respective blood flow. The amount passing through the liver is measured from the concentration of the indicator in the hepatic venous blood and the blood flow through the liver. Some indicator recirculates to the portal vein by way of the arteries and is measured in the femoral artery. When absorption is complete the total amount of the indicator absorbed may be determined from the product of its concentration and its volume of distribution.

During the time required to reach equilibrium, the difference between the amount of radioactivity leaving the splanchnic bed and the amount entering should be equal to the effective radioactivity

* Present addresses: Dr. Iber, The Johns Hopkins Hospital, Baltimore, Md. ; Dr. Kerr, Royal Victoria Infirmary, Newcastle upon Tyne, England; Dr. Dölle, University-Krankenhaus Eppendorf, Hamburg, Germany: Dr. Sherlock, Royal Free Hospital, Grays Inn Road, London, England.



Fig. 1. Diagram of the experimental method. HISA is injected into the spleen and may reach the peripheral circulation by passing through the liver or through the collateral vessels. HISA passing through the liver may be measured by means of a hepatic vein catheter.

injected minus the residual radioactivity in the splanchnic plasma. The radioactivity leaving the splanchnic bed is that leaving by the hepatic vein plus that leaving by the collaterals.

\section{METHOD}

Assumptions. The major assumptions of the method are listed below in the same order in which they appear in the method and the evaluation of the data. Continuous sampling from the hepatic vein must extend throughout the period of absorption of indicator from the spleen. The indicator must mix completely with the blood in the splenic vein before the first branching point and with all blood subsequently entering the splenic vein. The sampling from the hepatic vein must be uniform and must not alter the blood flow in the hepatic vein. The sulfobromophthalein (BSP) technique must measure blood flow in the hepatic vein. Recirculated indicator must take the same route as injected indicator and this route must not vary during the study. The time of circulation from heart to hepatic vein is the same as circulation from heart to femoral artery. The splanchnic blood volume may be estimated from values in the literature. The flow of significant indicator through collateral circulation 
is slow compared with the flow through the hepatic vein. The total blood flow through the liver must not change during the procedure.

A preliminary investigation was carried out to test the suitability of human iodinated serum albumin (HISA) as an indicator. The HISA was prepared by the technique of McFarlane (6) and had an activity of 5 to 25 $\mu \mathrm{c}$ per $\mathrm{ml}$. A measured dose of HISA in 1 to $2 \mathrm{ml}$ was injected into the spleen and samples were taken from a peripheral artery or vein for 20 minutes to determine concentration. The plasma volume was determined with Evans blue. Eight patients with splenomegaly were studied; 6 had portal hypertension. The time necessary to reach a constant concentration was from 2 to 3.5 minutes (mean, 2.8 minutes) and the constant concentration was 94 to 106 per cent (mean, 98 per cent) of that predicted if the injected dose were distributed into the Evans blue volume. It was concluded that HISA in small volumes was rapidly and almost completely absorbed from the injection site in the spleen and that its rate of appearance in the circulation could be determined if frequent samples were taken in the first 4 minutes. No significant leakage from the circulation occurred within the period of study.

Measurement of collateral blood flow was attempted in 12 patients after an overnight fast and without premedication. On the day prior to the test, the rate of removal from the blood of BSP ( $5 \mathrm{mg}$ per $\mathrm{kg}$ ) was determined. The volume of distribution (plasma volume), the rate of removal of BSP and the rate of constant infusion necessary for measurement of hepatic blood flow were calculated (7). Samples were analyzed for BSP by the technique of Seligson, Marino and Dodson (8).

On the morning of the test a no. 9 radiopaque catheter was introduced into a right hepatic vein and the wedged hepatic vein pressure recorded. All pressures were expressed in millimeters $\mathrm{Hg}$ above the measured right auricular pressure and were obtained with a Müller-Shillingford photoelectric transducer and recorder manufactured by the Cambridge Instrument Co. (9). Normal values for wedged hepatic vein pressure are less than 12 $\mathrm{mm} \mathrm{Hg} \mathrm{(10-13).}$

An indwelling needle was inserted into a femoral artery and a priming dose of BSP, followed by a constant infusion of BSP, was administered into the arm vein. Paired samples of arterial and hepatic venous blood were withdrawn at intervals for determination of hepatic plasma flow (14).

Intrasplenic pressure was then measured (15) and, with the needle in position, 2 to $20 \mu \mathrm{c}$ of HISA in 1 to $2 \mathrm{ml}$ was injected deeply into the spleen. Normal intrasplenic pressure is less than $14 \mathrm{~mm} \mathrm{Hg}(15,16)$. Starting at the time of injection, continuous simultaneous manual withdrawal of blood was made from the hepatic vein catheter and from the femoral artery at the rate of $1 \mathrm{ml}$ each 5 seconds. Syringes were usually changed at 30 -second intervals during the first 1.5 minutes and at 1 minute intervals thereafter for 3 additional minutes. A single sample was taken from each site atter $10 \mathrm{~min}$ -

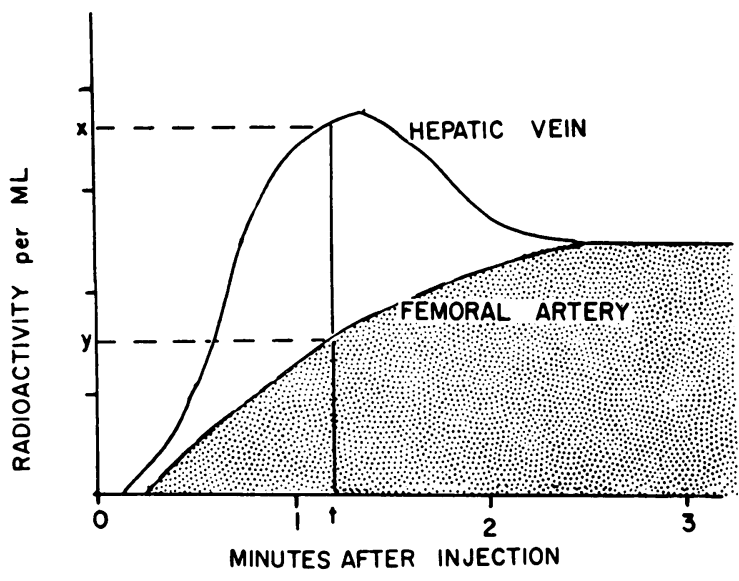

Fig. 2. Hypothetical RADioACtivity of Femoral ARTERIAL AND HEPATIC VENOUS PLASMA AFTER INJECTION OF HISA INTO SPLEEN AT ZERO TIME. Until absorption is complete, the hepatic venous values are greater than the femoral arterial values.

utes. Samples were heparinized and BSP and radioactivity were measured in plasma. Counting was performed in a well-scintillation counter that measured $1.2 \times 10^{6}$ cpm per $\mu \mathrm{c}$ of iodine ${ }^{131}$. Plasma volume was measured with Evans blue dye in 4 subjects. The volume of distribution for BSP was used in the remainder.

Calculation. A curve was constructed of the radioactivity measurements in the hepatic venous and femoral arterial plasma, such as is shown in the hypothetical example of Figure 2. The upper heavy line represents hepatic venous radioactivity and the lower line (at the upper border of the stippled area) represents femoral arterial radioactivity, which is assumed equal to the levels in recirculated arterial plasma leaving the splanchnic circulation at the same time. When the arterial and venous lines join, the absorption of radioactivity is complete and there is equilibrium.

Calculation is based on the premise that during the time required to reach equilibrium, the difference between the amount of radioactivity leaving the splanchnic bed and the amount entering it should be equal to the effective radioactivity injected minus the residual radioactivity in the splanchnic plasma. The radioactivity leaving the splanchnic bed is that leaving by the hepatic veins $\left(Q_{\mathrm{hv}}\right)$ plus that leaving by the collaterals $\left(Q_{\text {col1 }}\right)$. The radioactivity entering the splanchnic bed $\left(Q_{\mathrm{fa}}\right)$ is the product of the femoral arterial concentration and the hepatic plasma flow $\left(F_{h v}\right)$. The effective radioactivity injected is the product of the equilibrium concentration $\left(C_{E}\right)$ and the plasma volume $\left(V_{P}\right)$. The residual splanchnic radioactivity is the product of the splanchnic plasma volume $\left(\mathrm{V}_{\mathrm{spl}}\right)$ and the equilibrium concentration $\left(C_{E}\right)$. The splanchnic bed is defined as those blood vessels that drain through the hepatic vein.

Then :

$$
\begin{aligned}
& Q_{\mathrm{hv}}+Q_{\mathrm{coll}}-Q_{\mathrm{fa}}=\mathrm{V}_{\mathrm{P}} C_{\mathrm{E}}-\mathrm{V}_{\mathrm{spl}} C_{\mathbf{E}} \\
& Q_{\mathrm{coll}}=\left(\mathrm{V}_{\mathrm{P}}-\mathrm{V}_{\mathrm{spl}}\right) \mathrm{C}_{\mathbf{E}}+Q_{\mathrm{fa}}-Q_{\mathrm{hv}} .
\end{aligned}
$$


TABLE I

Sample calculation

\begin{tabular}{ccccc}
\hline \hline Time & Arterial & Venous & Difference & Area \\
\hline $\min$ & & $10^{3} \mathrm{cpm} / \mathrm{ml}$ & & $\left(10^{3} \mathrm{~min}\right)(\mathrm{cpm} / \mathrm{ml})$ \\
$0-0.5$ & 4.6 & 11.8 & 7.2 & 3.6 \\
$0.5-1.0$ & 16.2 & 21.9 & 5.7 & 2.8 \\
$1.0-1.5$ & 11.6 & 19.8 & 8.2 & 4.1 \\
$1.5-2.0$ & 12.5 & 16.4 & 3.9 & 1.9 \\
$2.0-3.0$ & 11.6 & 12.5 & 0.9 & 0.9 \\
$3.0-4.0$ & 11.6 & 11.6 & \multicolumn{2}{c}{ Total $13.3 \times 10^{3}$} \\
& & & & \\
\hline
\end{tabular}

In Equation 1, $\mathrm{V}_{\mathrm{spl}}$ is assumed to be either 21 per cent of $V_{P}$ or is calculated from Equation 1, assuming $Q_{\text {col1 }}$ to be zero. $V_{P}$ and $C_{E}$ are determined directly from the data. $Q_{f_{a}}-Q_{h v}$ is obtained from the plot of the data as shown in Figure 2. In Figure 2, at any time (e.g., t) the vertical distance $(x)$ to the upper line represents radioactivity per milliliter of plasma passing through the hepatic veins, and the vertical distance $(y)$ to the lower line represents the portion of radioactivity derived from recirculated splanchnic arterial plasma. The distance $(\mathrm{x}-\mathrm{y})$ between the lines therefore represents the radioactivity per milliliter of hepatic vein plasma absorbed from the spleen. The radioactivity absorbed from the spleen via the hepatic veins between time $t$ and time $t+d t$ is therefore $F_{h v}(x-y) d t$ microcuries. The total radioactivity absorbed through the hepatic veins or the unknown quantity $Q_{\mathrm{hv}}-Q_{\mathrm{fa}}$ in Equation 1 becomes

$$
Q_{\mathrm{hv}}-Q_{\mathrm{fa}}=F_{\mathrm{hv}} \int_{0}^{\mathrm{T}}(\mathrm{x}-\mathrm{y}) \mathrm{dt} \text {. }
$$

Sample calculation: A sample calculation (Table I) will better illustrate this manipulation. Table II contains pertinent clinical and experimental data.

The experimental hepatic venous and femoral arterial levels from Patient 1 are illustrated in Figure 3 . Each

\section{SPLENOMEGALY}



Fig. 3. Plasma concentration data from Patient 1. Each horizontal block is a single sample of plasma collected by continuous sampling during the time indicated by the widths of the block. Total absorption is calculated by dividing the dose given intrasplenically by the measured blood volume. vertical bar represents a single sample collected by continuous constant withdrawal of blood during the time indicated on the abscissa. $Q_{h v}-Q_{\mathrm{fa}}$ is calculated from Equation 2. The value of the integral is obtained graphically by determining the area between the hepatic venous and the femoral arterial curves. Thus, in Figure 3 the value of the clear area for each block until equilibrium is listed.

This value when multiplied by the plasma flow (Table II) in milliliters per minute yields $Q_{\mathrm{hv}}-Q_{\mathrm{fa}}\left(13.3 \times 10^{3}\right.$ $\times 665=8.72 \times 10^{\circ}$ ). If the concentration in the femoral artery is greater than that in the hepatic vein the difference is calculated as zero; negative values are not considered. If $Q_{\text {coll }}$ is assumed to be zero, the value of $\mathrm{V}_{\text {spl }}$ may be calculated directly from Equation 1. This value is recorded for each patient in Table II as the calculated $V_{s p 1}$ expressed as a percentage of $V_{P}$.

For this patient the calculation is as follows : Equation 1:

$$
\begin{aligned}
& \frac{V_{s p l}}{V_{P}}=\frac{Q_{f a}-Q_{h v}-Q_{c o l l}+V_{P} C_{E}}{V_{P} C_{E}} \\
&=1-\frac{8.82 \times 10^{6}}{11.2 \times 10^{6}}=0.22 .
\end{aligned}
$$

Thus the $\mathrm{V}_{\mathrm{spl}}$ is 22 per cent of $\mathrm{V}_{\mathrm{P}}$.

In order to calculate collateral flow, $\mathrm{V}_{\mathrm{spl}}$ is assumed to be 21 per cent of $V_{P}$, and Equation 1 is rearranged to yield: Equation 1:

$$
\begin{aligned}
& \frac{Q_{\text {coll }}}{Q_{\text {coll }}+Q_{\mathrm{hv}}-Q_{\mathrm{fa}}}=\frac{0.79 \mathrm{~V}_{\mathrm{P}} C_{\mathrm{E}}+Q_{\mathrm{fa}}-Q_{\mathrm{hv}}}{0.79 \mathrm{~V}_{\mathrm{P}} C_{\mathrm{E}}} \\
& =1-\frac{8.72 \times 10^{6}}{8.84 \times 10^{6}}=0.01 .
\end{aligned}
$$

Thus the fraction of portal blood flowing through collaterals is 1 per cent.

\section{RESULTS}

The clinical details, splenographic findings and calculation of collateral flow in 12 patients are given in Table II. Patients 1 to 3 had no clinical evidence of collateral blood flow. The calculated collateral blood flows are very low, in agreement with the clinical data. The calculated splanchnic plasma volumes of 22,18 and 20 per cent of the total were in excellent agreement with values from the literature (17-19). Patient 4 had had ascites on admission but at the time of study the ascites had disappeared. There was no clinical evidence of collateral vessels, but portal hypertension was present. The calculated splanchnic plasma volume was 38 per cent and the calculated collateral flow was 22 per cent.

Patients 5 to 7 had cirrhosis with definite clinical evidence of collateral circulation. The calculated splanchnic plasma volumes were 36,55 and 85 per cent of the plasma volume, respectively. 


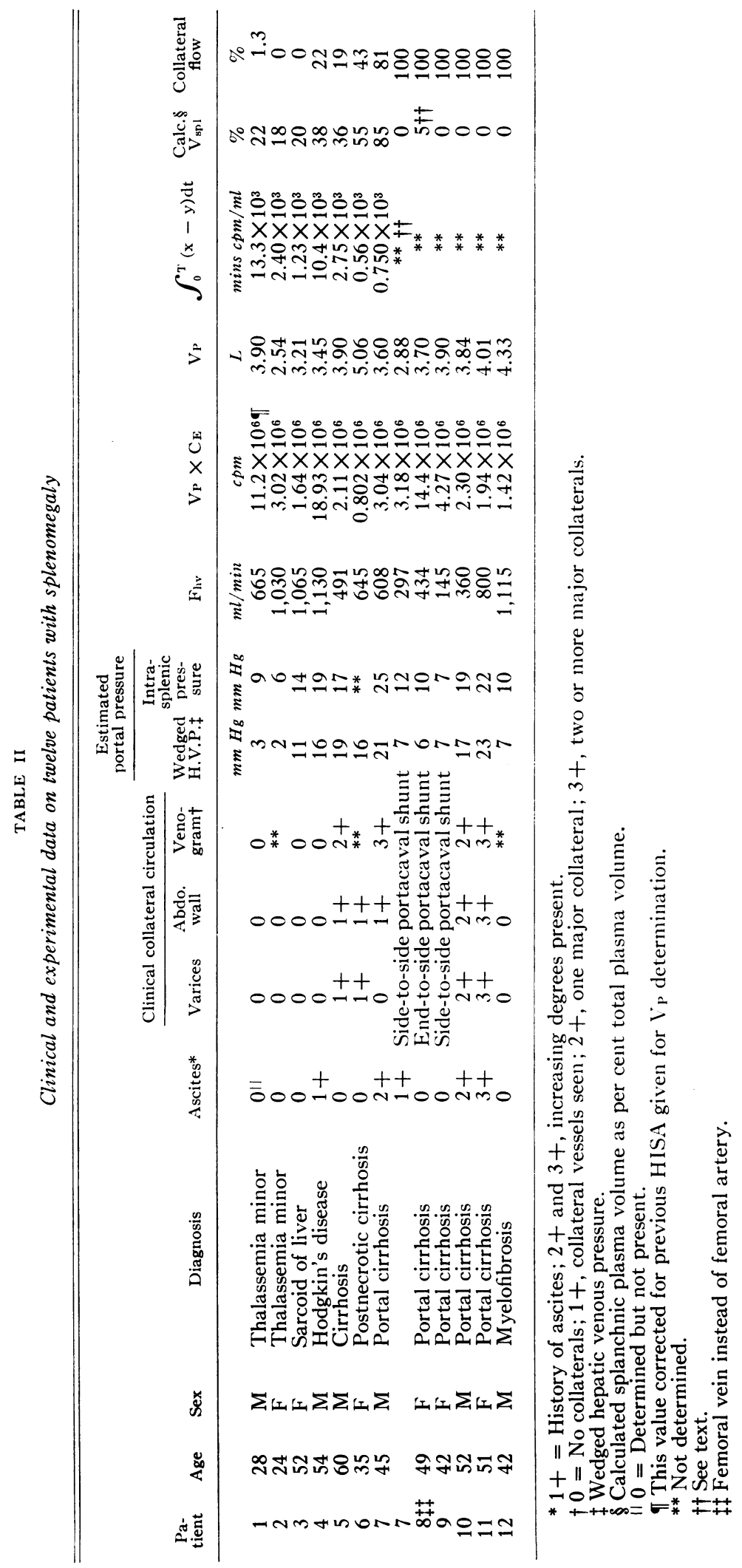




\section{CIRRHOSIS}

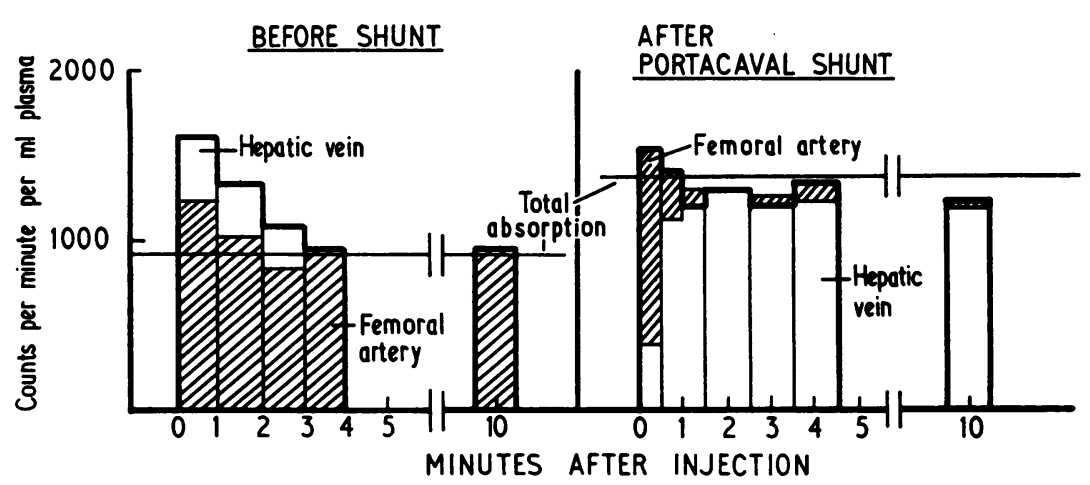

Fig. 4. Two similar studies on Patient 7. In the first study the hepatic venous values were greater than those for the femoral artery. This was reversed after the shunt.

The corresponding calculated collateral flows were 19, 43 and 81 per cent. The results in a patient with collateral circulation (Patient 7) are illustrated in Figure 4; this patient was studied before and after side-to-side portacaval shunt. In the study before shunting, the hepatic venous samples prior to equilibrium contained more HISA than did the femoral arterial samples. The area between the curves was smaller than in Figure 3, indicating that a smaller proportion of the HISA was absorbed via the liver than in the patient without collaterals. The calculated collateral flow was 81 per cent.

In Patient 7, following a side-to-side portacaval shunt, the arterial blood contained more radioactivity than did the hepatic venous blood, indicating that radioactivity arrived at the liver first through the hepatic artery. Therefore, the calculated collateral flow was 100 per cent. Two other patients were studied after surgical portacaval shunts. In Patient 8 the shunt was end-toside and in Patient 9, side-to-side; in both, the peripheral blood contained more radioactivity than did the hepatic venous blood until equilibrium was reached. The patients with past or present ascites had a higher calculated collateral flow than had those without ascites.

In Patients 10, 11 and 12 the results were quite unexpected. Both 10 and 11 had cirrhosis with ascites and clinical evidence of marked collateral circulation. In both, the radioactivity in the arterial blood was higher than that in the hepatic venous blood. The peculiar finding was also pres- ent in Patient 12 who had no clinical evidence of collateral circulation.

\section{DISCUSSION}

The important assumptions used in calculating the collateral flow are listed in the Method section. Several of these deserve comment.

The absorbed HISA must mix completely in the splenic vein before the first effluent venous branch is reached and at all points where new blood is added. Though mixing is probably complete in the small vessels and in splenic and portal veins of normal size, the increased diameter of the vessels and the reduced blood flow in portal hypertension make streamlining possible and even probable (20). This has been frequently observed in splenic venograms, particularly at the entrance of the inferior and superior mesenteric veins (16, 21).

The splanchnic plasma volume cannot be measured in the presence of collateral circulation (17); it must therefore be estimated in order to calculate the collateral flow. The value has been determined in subjects without liver disease. A mean splanchnic plasma volume of 21 per cent of the plasma volume was found $(17-19)$; the variation, however, was large. The portal vein and its branches are dilated in portal hypertension; their capacity is therefore enlarged. In the presence of a collateral circulation, part of the portal vein blood drains through the liver and part drains through the collateral vessels. The splanchnic plasma volume used in Equation 1 is defined as 
the volume of plasma contained in the vessels draining through the liver. By this definition the fraction of splanchnic blood in the collaterals must not be included. Even though the capacity of the portal bed is enlarged, the value of 21 per cent is still reasonable.

The radioactivity in the femoral artery does not exactly represent the radioactivity in recirculated arterial blood. Recirculated radioactivity mixed in the splanchnic bed, and sampled as hepatic venous blood, left the aorta some time earlier (about 12 seconds) than the blood sampled simultaneously at the femoral artery. In portal hypertension the circulation time of blood from the aorta to the hepatic veins is prolonged and therefore this error is greater. This prolongation in portal hypertension is approximately 20 seconds (20, 22-24). Applying this correction to the data decreases the calculated collateral flow by an average of about 8 per cent. This error assumes particular importance if some radioactivity reaches the peripheral circulation through a collateral vessel much earlier than it would through the liver, possibly by-passing the pulmonary circulation. Under these circumstances the radioactivity in the peripheral arterial blood is much higher than the simultaneously sampled recirculated arterial blood mixed in the portal bed and sampled at the hepatic vein. Such an error leads to overestimation of the collateral flow.

A change in blood flow of 5 to 10 per cent following the intrasplenic injection might be missed in the short time of sampling by the BSP technique.

In Patients 1 to 3 the calculated splanchnic plasma volumes were in very good agreement with the values given in the literature. In $\mathrm{Pa}$ tients 4 and 5 the calculated splanchnic plasma volumes of 36 and 38 per cent were just outside the range of normal in patients without collaterals. Therefore, such values may represent the splanchnic plasma volume. On the other hand, both patients showed clinical evidence of collateral vessels, suggesting that calculated $\mathrm{V}_{\text {spl }}$ is too high.

In Patients 6 and 7 the calculated values of 55 and 85 per cent for the splanchnic plasma volume are obtained, assuming no collateral flow. Such high values for splanchnic plasma volume are extremely unlikely; collateral flow must be present.

In patients with end-to-side portacaval shunts, radioactive blood reaches the liver only through the hepatic artery, and all of this blood leaves the liver through the hepatic veins without bypass. The volume of blood in all vessels from the hepatic artery to the hepatic veins may be calculated (17). In Patient 8 the value for this plasma volume of 5 per cent was obtained. This agrees adequately with the figure of 6 per cent for this plasma volume found in dogs (25).

Patients 10 to 12 had calculated collateral flows of 100 per cent. This is unexpected in patients without surgical portacaval shunts. In all three patients the radioactivity was higher in the peripheral artery than in the hepatic vein. The highest values of radioactivity in the peripheral artery in these patients appeared in the first half minute after sampling (as in Figure 4 after shunt). The highest values of radioactivity in the artery in all other patients appeared later, just after the end of the first minute on the average. The rate of appearance of radioactivity in the hepatic venous blood was about the same as the rate in the other patients. These findings suggest a high blood flow of short circulation time through collateral vessels. Such a shunt has been observed by Reichman, Davis, Storaasli and Gorlin (26) and may represent the shunt from the portal vein to the left side of the heart demonstrated in the anatomical studies in cirrhotic patients of Abelmann and coworkers (27). Such a mechanism would produce a high concentration of HISA in a very small part of the peripheral circulation (left heart and aorta) before this portion of radioactive material could appear in the hepatic vein by recirculation. $\mathrm{Pa}$ tient 11 showed oxygen-refractory desaturation of the arterial blood which is evidence that venous blood bypasses the lung.

Another explanation of these findings would be a misplaced catheter in the inferior vena cava. The removal of BSP, however, showed that the catheter remained in the hepatic vein. A further explanation could be the injection of HISA into the muscle or peritoneal cavity. The recording of the intrasplenic pressure in all three patients and the nearly complete absorption of injected HISA in two of the three indicates that the needle was in the spleen. In Patient 12, however, only one-third of the administered dose appeared in the circulation in ten minutes. Equilibrium was reached in three minutes. Megakaryocytes were 
seen in a smear made from the needle just prior to injection, confirming that the needle was in the spleen. There is no satisfactory explanation of the findings in Patient 12.

\section{SUMMARY}

A method for the estimation of blood flow through the collateral vessels of the portal vein is described. The concentration of iodinated albumin injected into the spleen appearing at the hepatic vein and in the peripheral circulation is measured, and from the difference the collateral flow is estimated. The results of this method correlate with clinical and radiological evidence of collateral circulation. Some patients show very rapid circulation of the iodinated albumin through collateral vessels which makes application of the method impossible.

\section{REFERENCES}

1. Abeatici, S., and Campi, L. Sur les possibilités de l'angiographie hépatique; la visualisation du système portal; recherches expérimentales. Acta radiol. (Stockh.) 1951, 36, 383.

2. Atkinson, M., Barnett, E., Sherlock, S., and Steiner, R. E. The clinical investigation of the portal circulation, with special reference to portal venography. Quart. J. Med. 1955, 24, 77.

3. Bergstrand, I., and Ekman, C-A. Portal circulation in portal hypertension. Acta radiol. (Stockh.) $1957,47,1$.

4. Leger, L. H. Spléno-portographie. Paris, Masson, 1955.

5. Summerskill, W. H. J., Davidson, E. A., Sherlock, S., and Steiner, R. E. The neuropsychiatric syndrome associated with hepatic cirrhosis and an extensive portal collateral circulation. Quart. J. Med. 1956, 25, 245.

6. McFarlane, A. S. Labelling of plasma proteins with radioactive iodine. Biochem. J. 1956, 62, 135.

7. Iber, F. L., Craigie, A., Dölle, W., and Sherlock, S. Comparison of BSP and galactose techniques for liver blood flow. In preparation.

8. Seligson, D., Marino, J., and Dodson, E. Determination of sulfobromophthalein in serum. Clin. Chem. 1957, 3, 638.

9. Müller, O., and Shillingford, J. P. A manometer for differential and single pressure measurements. J. Physiol. (Lond.) 1954, 127, 2-P.

10. Myers, J. D., and Taylor, W. J. An estimation of portal venous pressure by occlusive catheterization of an hepatic venule (abstract). J. clin. Invest. 1951, 30, 662.

11. Taylor, W. J., and Myers, J. D. Occlusive hepatic venous catheterization in the study of normal liver, cirrhosis of the liver and non-cirrhotic portal hypertension. Circulation 1956, 13, 368.

12. Krook, H. Circulatory studies in liver cirrhosis. Acta med. scand. 1956, 156, suppl. 318.

13. Reynolds, T. B., Redeker, A. G., and Geller, H. M. Wedged hepatic venous pressure: A clinical evaluation. Amer. J. Med. 1957, 22, 341.

14. Bradley, S. E., Ingelfinger, F. J., Bradley, G. P., and Curry, J. J. The estimation of hepatic blood flow in man. J. clin. Invest. 1945, 24, 890.

15. Atkinson, M., and Sherlock, S. Intrasplenic pressure as index of portal venous pressure. Lancet 1954, 1, 1325.

16. Turner, M. D., Sherlock, S., and Steiner, R. E. Splenic venography and intersplenic pressure measurement in the clinical investigation of the portal venous system. Amer. J. Med. 1957, 23, 846.

17. Bradley, S. E., Marks, P. A., Reynell, P. C., and Meltzer, J. The circulating splanchnic blood volume in dog and man. Trans. Ass. Amer. Phycns 1953, 66, 294.

18. Rapaport, E., Weisbart, M. H., and Levine, M. The splanchnic blood volume in congestive heart failure. Circulation 1958, 18, 581.

19. Wade, O. L., Combes, B., Childs, A. W., Wheeler, H. O., Cournand, A., and Bradley, S. E. The effect of exercise on the splanchnic blood flow and splanchnic blood volume in normal man. Clin. Sci. 1956, 15, 457.

20. Hunt, A. H. A Contribution to the Study of Portal Hypertension. Edinburgh, E. and S. Livingstone, 1958.

21. Leger, L. L'inversion du courant portal. Les fausses images d'obstacle à la circulation sur le tronc porte. Presse méd. 1956, 64, 1189.

22. Wheeler, H. O., Combes, B., Childs, A. W., and Bradley, S. E. Distribution of splanchnic circulation times in cirrhosis (abstract). J. clin. Invest. $1956,35,744$.

23. Wheeler, H. O., Combes, B., Childs, A. W., and Wade, O. L. The splanchnic circulation time. Trans. Ass. Amer. Phycns 1955, 68, 177.

24. Mena, I., Bennett, L. R., Kivel, R., Scallon, J., and Mellinkoff, S. M. Determination of cardioportal circulation time by external scintillation counting. Amer. J. dig. Dis. 1959, 4, 19.

25. Johnstone, F. R. C. Measurement of splanchnic blood volume in dogs. Amer. J. Physiol. 1956, $185,450$.

26. Reichman, S., Davis, W. D., Storaasli, J. P., and Gorlin, R. Measurement of hepatic blood flow by indicator dilution techniques. J. clin. Invest. 1958, 37, 1848.

27. Abelmann, W. H., Calabresi, P., Kramer, G., McNeely, W. F., and Gravallese, M. A. Arterial unsaturation, venous admixture, and portopulmonary anastomosis in patients with cirrhosis of the liver. J. clin. Invest. 1955, 34, 919. 